\title{
Silica-supported ionic liquid as highly efficient catalyst for one-pot synthesis of acenaphtho[1,2-b]furan compounds
}

\author{
REZA SANDAROOS $^{\mathrm{a}, *}$, MOHAMMAD TAGHI GOLDANI $^{\mathrm{b}, *}$ and SAMAN DAMAVANDI ${ }^{\mathrm{c}}$ \\ ${ }^{a}$ Department of Chemistry, Faculty of Science, University of Birjand, Birjand 9717851367, Iran \\ ${ }^{b}$ Department of Engineering, Birjand Branch, Islamic Azad University, Birjand 9717851367, Iran \\ ${ }^{c}$ Department of Chemistry, Sarvestan Branch, Islamic Azad University, Sarvestan 9717851367, Iran \\ e-mail: R_Sandaroos@yahoo.com; r_sandaroos@birjand.ac.i; mt.goldani@yahoo.com
}

MS received 16 September 2012; revised 20 January 2013; accepted 1 March 2013

\begin{abstract}
Some new derivatives of acenaphtho[1,2-b]furan have been synthesized efficiently by one-pot reaction of (acenaphthylen-1-yloxy)trimethylsilane, various aldehydes and isocyanides at the presence of silica-supported ionic liquid.
\end{abstract}

Keywords. One-pot synthesis; acenaphtho[1,2-b]furan; silica-supported ionic liquid.

\section{Introduction}

During the past years, the synthesis of pharmacologically active furans, were widely known in nature, ${ }^{1,2}$ have received much interest, probably, with the aim of preparing more biologically active derivatives of furans. ${ }^{3-7}$ Isocyanide-based multi component reactions emerged in 1990s by Passerini, ${ }^{8}$ are among the most important methods that have attracted more interest. ${ }^{9-14}$

As against traditional solvents, ionic liquids benefit from advantages such as undetectable vapour pressure, ease of being recycled, and their ability to dissolve many organic and inorganic substances and play specific tasks such as Brønsted acidic task-specific ionic liquids (BAILs). ${ }^{15}$ In this regard, ionic liquids possessing $\mathrm{HSO}_{4}^{-}$as counteranion, especially those have been immobilized on the solid surfaces, exhibit promising results in the area of catalysing reactions. ${ }^{16-20}$

In the previous studies, ${ }^{21,22}$ we reported catalystfree synthesis of acenaphtho [1,2-b]furan compounds employing isocyanide, aldehyde and silyl enol of acenaphthylen-1(2H)-one. However, chemical yields were not sustainable. Accordingly, in this study, we examined various ionic liquids to improve efficiency of our synthetic method. Among ionic liquids used, supported-ionic liquid (d) was found as the best choice and used for synthesis of compounds (7a-k)

*For correspondence (scheme 1). In each case, efficiencies of catalysed and uncatalysed reaction were compared (table 1).

\section{Experimental}

\subsection{Materials and methods}

Compounds (7c-g) are new and characterized by IR, ${ }^{1} \mathrm{HNMR},{ }^{13} \mathrm{CNMR}$ and elemental micro analysing. ${ }^{1} \mathrm{HNMR}$ spectra were recorded on a Bruker AQSAVANCE-400 $\mathrm{MHz}$ spectrometer using TMS as an internal standard $\left(\mathrm{CDCl}_{3}\right.$ solution). ${ }^{13} \mathrm{CNMR}$ spectra were recorded on a Bruker AQSAVANCE$125 \mathrm{MHz}$ spectrometer $\left(\mathrm{CDCl}_{3}\right.$ solution). IR spectra were recorded from $\mathrm{KBr}$ disk on the FT-IR Bruker Tensor. Elemental analyses were performed using a Heraeus CHN-O-Rapid analyzer. (acenaphthylen1-yloxy)trimethylsilane (1), supported and unsupported ionic liquids were prepared according to the literatures. ${ }^{23,24}$

\subsection{General procedure for preparation of acenaphtho[1,2-b]furan derivatives $(7 \boldsymbol{a}-\boldsymbol{k})$}

To a magnetically stirred mixture of aldehyde $(1 \mathrm{mmol})$ and catalyst $(0.01 \mathrm{mmol})$ in DMF $(40 \mathrm{~mL})$ at $0^{\circ} \mathrm{C}$ was drop-wise added to a solution of (acenaphthylen1-yloxy)trimethylsilane (1) $(1 \mathrm{mmol})$ in DMF (10 mL) over a $30 \mathrm{~min}$ period. Then, the mixture was allowed to warm to room temperature and treated with a solution of isocyanide $(1.0 \mathrm{mmol})$ in DMF $(10 \mathrm{~mL})$. The mixture was refluxed for $10 \mathrm{~h}$, cooled to room temperature, and filtered. The filtrate was extracted with dichloromethane 

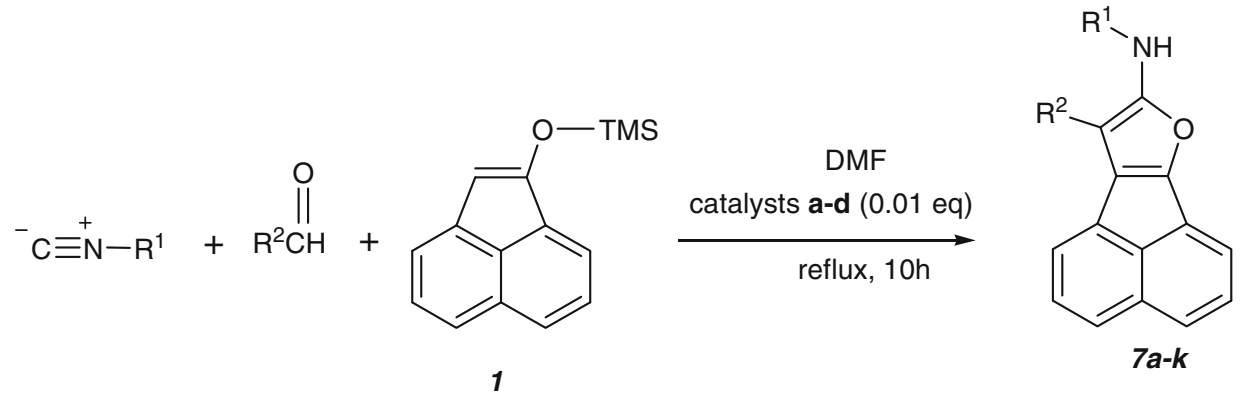

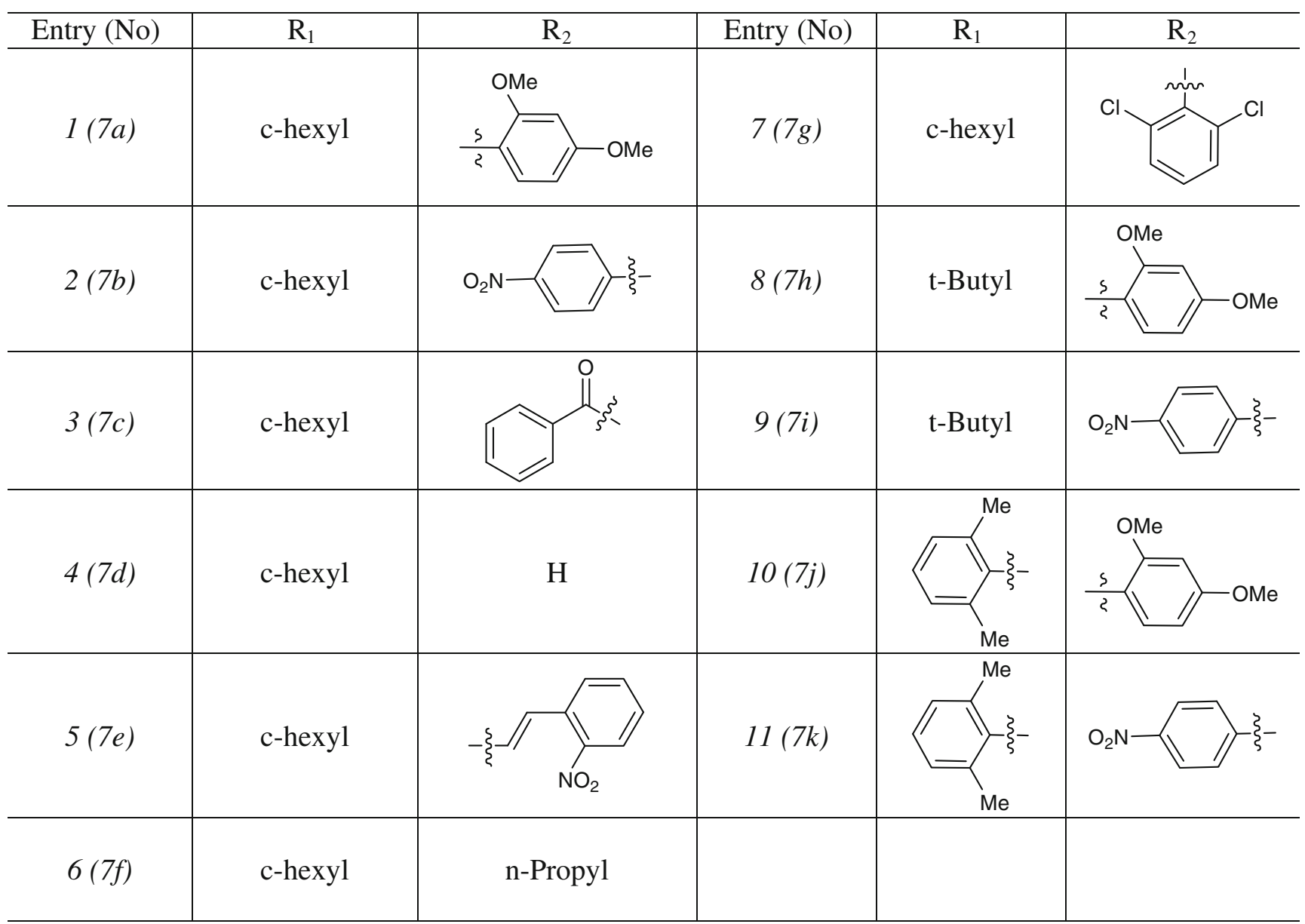



$\mathrm{X}=(\mathbf{a}): \mathrm{Cl} ;(\mathbf{b}): \mathrm{BF}_{3} ;(\mathbf{c}): \mathrm{HSO}_{4}$

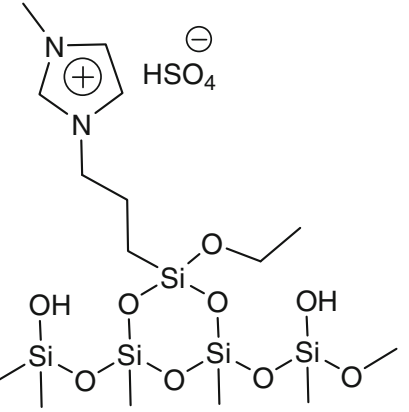

(d): [pmim $] \mathrm{HSO}_{4}-\mathrm{SiO}_{2}$

Scheme 1. Preparation of compounds $(7 \mathbf{a}-\mathbf{k})$ at the presence of ionic liquids $(\mathbf{a}-\mathbf{d})$. 
Table 1. Comparison of ionic liquid-catalysed and uncatalysed routs for preparation of compounds (7a-k).

\begin{tabular}{lccc}
\hline Entry & Compound & $\begin{array}{c}\text { Time }(\mathrm{h}) / \text { Yield }(\%)^{\mathrm{a}}, \\
\text { catalysed by catalyst (d) }\end{array}$ & $\begin{array}{c}\text { Time }(\mathrm{h}) / \text { Yield }(\%)^{\mathrm{a}} \\
\text { uncatalysed }\end{array}$ \\
\hline 1 & $\mathbf{7 a}$ & $10 / 97$ & $24 / 89^{22}$ \\
2 & $\mathbf{7 b}$ & $10 / 95$ & $24 / 93^{22}$ \\
3 & $\mathbf{7 c}$ & $10 / 94$ & $24 / 76$ \\
4 & $\mathbf{7 d}$ & $10 / 92$ & $24 / 81$ \\
5 & $\mathbf{7 e}$ & $10 / 91$ & $24 / 75$ \\
6 & $\mathbf{7}$ & $10 / 95$ & $24 / 82$ \\
7 & $\mathbf{7 g}$ & $10 / 92$ & $24 / 80$ \\
8 & $\mathbf{7}$ & $10 / 95$ & $24 / 74^{22}$ \\
9 & $\mathbf{7}$ & $10 / 93$ & $24 / 78^{22}$ \\
10 & $\mathbf{7 i}$ & $10 / 96$ & $24 / 82^{21}$ \\
11 & $\mathbf{7 j}$ & $10 / 94$ & $24 / 86^{21}$ \\
\hline
\end{tabular}

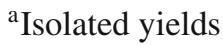

$(4 \times 15 \mathrm{~mL})$. After evaporation of solvent, the residue was washed with ether and crystallized from acetonitrile to give analytically pure product $(\mathbf{7 a - k})$.

2.2a N-cyclohexyl-9-(2,4-dimethoxyphenyl)acenaphtho[1, 2-blfuran-8-amine (7a): Brown powder (97\%); m.p. 195-200 ${ }^{\circ}$; FT-IR $(\mathrm{KBr})\left(\nu_{\max }, \mathrm{cm}^{-1}\right): 3355(\mathrm{~N}-\mathrm{H})$, $1230(\mathrm{C}-\mathrm{O}), 1200(\mathrm{C}-\mathrm{O}) ;{ }^{1} \mathrm{H}$ NMR $\left(400 \mathrm{MHz}, \mathrm{CDCl}_{3}\right)$ $\delta_{\mathrm{H}} 1.21-1.97\left(10 \mathrm{H}, \mathrm{m}, 5 \mathrm{CH}_{2}\right), 3.52(1 \mathrm{H}, \mathrm{m}, \mathrm{N}-\mathrm{CH})$, $3.75\left(3 \mathrm{H}, \mathrm{s}, \mathrm{OCH}_{3}\right), 3.81\left(3 \mathrm{H}, \mathrm{s},-\mathrm{OCH}_{3}\right), 6.43(1 \mathrm{H}$, bs, NH), 6.62-6.72 (2H-m, Ar-H), $7.23(1 \mathrm{H}, \mathrm{d}, \mathrm{Ar}-$ $\mathrm{H}), 7.63-7.81(6 \mathrm{H}, \mathrm{m}, \mathrm{Ar}-\mathrm{H}) ;{ }^{13} \mathrm{C}$ NMR $(100 \mathrm{MHz}$, $\left.\mathrm{CDCl}_{3}\right) \delta_{\mathrm{C}} 22.6,28.7,31.5,49.6,55.9,56.2,100.9$, 107.1, 109, 117.8, 125.5, 127.3, 127.6, 128, 128.3, 129, 129.5, 133.5, 137.7, 139, 147.2, 158.4, 161.7; Anal. Calcd. for $\mathrm{C}_{28} \mathrm{H}_{27} \mathrm{NO}_{3}$ : C, 79.03; H, 6.40; N, 3.29\%. Found: C, 78.84; H, 6.35; N, 3.23\%.

2.2b N-cyclohexyl-9-(4-nitrophenyl)acenaphtho[1,2b]furan-8-amine (7b): Brown powder (95\%); m.p. 209-214 ${ }^{\circ} \mathrm{C}$; FT-IR $(\mathrm{KBr})\left(\nu_{\max }, \mathrm{cm}^{-1}\right): 3281(\mathrm{~N}-\mathrm{H})$, $1541(\mathrm{~N}-\mathrm{O}), 1360(\mathrm{~N}-\mathrm{O}) ;{ }^{1} \mathrm{H}$ NMR $\left(400 \mathrm{MHz}, \mathrm{CDCl}_{3}\right)$ $\delta_{\mathrm{H}} 1.24-2.09\left(10 \mathrm{H}, \mathrm{m}, 5 \mathrm{CH}_{2}\right), 3.60(1 \mathrm{H}, \mathrm{m}, \mathrm{N}-\mathrm{CH})$, $4.92(1 \mathrm{H}, \mathrm{bs}, \mathrm{NH}), 7.65-7.86(8 \mathrm{H}, \mathrm{m}, \mathrm{Ar}-\mathrm{H}), 8.35$ $(2 \mathrm{H}, \mathrm{dd}, \mathrm{Ar}-\mathrm{H}) ;{ }^{13} \mathrm{C}$ NMR $\left(100 \mathrm{MHz}, \mathrm{CDCl}_{3}\right) \delta_{\mathrm{C}} 24.5$, 26.1, 30.5, 30.8, 33.2, 51.3, 110.7, 121.4, 125.1, 125.7, $127.6,127.9$, 128.7, 129.1, 129.6, 130.3, 132.7, 135.1, 135.9, 137.4, 139.3, 141.5, 149.3, 154.8, 159.7, 175.4; Anal. Calcd. for $\mathrm{C}_{26} \mathrm{H}_{22} \mathrm{~N}_{2} \mathrm{O}_{3}: \mathrm{C}, 76.08 ; \mathrm{H}, 5.40 ; \mathrm{N}$, $6.82 \%$. Found: C, 75.91; H, 5.34; N, 6.76\%.

2.2c (8-(Cyclohexylamino)acenaphtho[1,2-b]furan9-yl)(phenyl)methanone (7c): Brown powder (94\%); m.p. $181-184^{\circ} \mathrm{C}$; FT-IR $(\mathrm{KBr})\left(v_{\max }, \mathrm{cm}^{-1}\right): 3350$ $(\mathrm{N}-\mathrm{H}), 1690(\mathrm{C}=\mathrm{O}) ;{ }^{1} \mathrm{H}$ NMR $\left(400 \mathrm{MHz}, \mathrm{CDCl}_{3}\right) \delta_{\mathrm{H}}$
1.20-2.01 (10H, m, 5CH $), 3.53(1 \mathrm{H}, \mathrm{m}, \mathrm{N}-\mathrm{CH}), 6.16$ (1H, bs, NH), 7.70-7.85 (5H, m, Ar-H), 7.90-8.04 (6H, m, Ar-H); ${ }^{13} \mathrm{C}$ NMR $\left(100 \mathrm{MHz}, \mathrm{CDCl}_{3}\right) \delta_{\mathrm{C}} 20.6,26.6$, $33.9,47.1,109.7,120.2,124.3,125.7,127.2,127.9$, 128.1, 128.5, 129.5, 133.1, 133.7, 135.1, 139.2, 149.3, 151.8, 190.7; Anal. Calcd. for $\mathrm{C}_{27} \mathrm{H}_{23} \mathrm{NO}_{2}$ : C, 82.42; H, $5.89 ; \mathrm{N}, 3.56 \%$. Found: C, 82.30; H, 5.81; N, 3.52\%.

2.2d N-cyclohexylacenaphtho[1,2-b]furan-8-amine $(7 \boldsymbol{d})$ : Brown powder $(92 \%)$; m.p. $161-163^{\circ} \mathrm{C}$; FT-IR (KBr) $\left(v_{\max }, \mathrm{cm}^{-1}\right): 3364(\mathrm{~N}-\mathrm{H}) ;{ }^{1} \mathrm{H}$ NMR $\left(400 \mathrm{MHz}, \mathrm{CDCl}_{3}\right)$ $\delta_{\mathrm{H}} 1.22-2.05\left(10 \mathrm{H}, \mathrm{m}, 5 \mathrm{CH}_{2}\right), 3.51(1 \mathrm{H}, \mathrm{m}, \mathrm{N}-\mathrm{CH})$, $4.76(1 \mathrm{H}, \mathrm{s},=\mathrm{CH}-), 6.09(1 \mathrm{H}, \mathrm{bs}, \mathrm{NH}), 7.78(2 \mathrm{H}, \mathrm{dd}$, Ar-H), 7.85-7.93 (4H, m, Ar-H); ${ }^{13} \mathrm{C}$ NMR $(100 \mathrm{MHz}$, $\left.\mathrm{CDCl}_{3}\right) \delta_{\mathrm{C}} 24.3,29.0,33.6,49.3,104.8,113.4,127.3$, 129.5, 129.9, 130.4, 131.7, 135.1, 138.9, 145.3, 150.2; Anal. Calcd. for $\mathrm{C}_{20} \mathrm{H}_{19} \mathrm{NO}$ : C, 83.01; H, 6.62; N, 4.84\%. Found: C, 82.95; H, 6.60; N, 4.81\%.

2.2e 9-(2-Nitrostyryl)-N-cyclohexylacenaphtho[1,2b]furan-8-amine (7e): Brown powder (91\%); m.p. 189-192 ${ }^{\circ}$ C; FT-IR $(\mathrm{KBr})\left(\nu_{\max }, \mathrm{cm}^{-1}\right): 3363(\mathrm{~N}-\mathrm{H})$, $1535(\mathrm{~N}-\mathrm{O}), 1354(\mathrm{~N}-\mathrm{O}) ;{ }^{1} \mathrm{H}$ NMR $\left(400 \mathrm{MHz}, \mathrm{CDCl}_{3}\right)$ $\delta_{\mathrm{H}} 1.29-2.01\left(10 \mathrm{H}, \mathrm{m}, 5 \mathrm{CH}_{2}\right), 3.53(1 \mathrm{H}, \mathrm{m}, \mathrm{N}-\mathrm{CH})$, $6.05(1 \mathrm{H}, \mathrm{bs}, \mathrm{NH}), 7.16(1 \mathrm{H}, \mathrm{d}, \mathrm{J}=12.4 \mathrm{~Hz}, \mathrm{ArC}=\mathrm{H})$, $7.65(1 \mathrm{H}, \mathrm{d}, \mathrm{J}=12.4 \mathrm{~Hz}, \mathrm{ArC}=\mathrm{CH}), 7.76-7.80(5 \mathrm{H}, \mathrm{m}$, Ar-H), 8.01-8.12 (4H, m, Ar-H), 8.40 (1H, dd, Ar-H); ${ }^{13} \mathrm{C}$ NMR $\left(100 \mathrm{MHz}, \mathrm{CDCl}_{3}\right) \delta_{\mathrm{C}} 26.2,31.6,35.1,53.3$, 111.6, 124.6, 128.6, 129.1, 130.9, 131.6, 131.8, 132.2, $132.5,133.1,134.2,137.6,137.7,139.3,141.9,143.2$, 150.3, 151.7; Anal. Calcd. for $\mathrm{C}_{28} \mathrm{H}_{24} \mathrm{~N}_{2} \mathrm{O}_{3}$ : C, 77.04; H, 5.54; N, 6.42\%. Found: C, 76.76; H, 5.47; N, 6.35\%.

$2.2 \mathrm{f} N$-cyclohexyl-9-propylacenaphtho[1,2-b]furan8-amine (7f): Brown powder (95\%); m.p. $174-176^{\circ} \mathrm{C}$; 
FT-IR $(\mathrm{KBr})\left(v_{\max }, \mathrm{cm}^{-1}\right): 3360(\mathrm{~N}-\mathrm{H}) ;{ }^{1} \mathrm{H}$ NMR $\left(400 \mathrm{MHz}, \mathrm{CDCl}_{3}\right) \delta_{\mathrm{H}} 0.92(3 \mathrm{H}, \mathrm{t}, \mathrm{J}=7.8 \mathrm{~Hz}$, $\left.\mathrm{CH}_{3}-\mathrm{C}-\mathrm{C}\right), 1.25-2.11\left(10 \mathrm{H}, \mathrm{m}, 5 \mathrm{CH}_{2}\right), 1.85(2 \mathrm{H}, \mathrm{m}$, $\left.\mathrm{C}-\mathrm{CH}_{2}-\mathrm{C}\right), 2.70\left(2 \mathrm{H}, \mathrm{t}, \mathrm{J}=7.8 \mathrm{~Hz}, \mathrm{C}-\mathrm{CH}_{2}-\mathrm{C}\right), 3.48$ $(1 \mathrm{H}, \mathrm{m}, \mathrm{N}-\mathrm{CH}), 5.06(1 \mathrm{H}, \mathrm{bs}, \mathrm{NH}), 7.69(2 \mathrm{H}, \mathrm{dd}, \mathrm{Ar}-$ $\mathrm{H}), 7.82-7.92(4 \mathrm{H}, \mathrm{m}, \mathrm{Ar}-\mathrm{H}) ;{ }^{13} \mathrm{C}$ NMR $(100 \mathrm{MHz}$, $\left.\mathrm{CDCl}_{3}\right) \delta_{\mathrm{C}} 17.1,20.3,26.7,28.6,30.1,33.6,51.7$, 113.1, 121.1, 127.6, 129.9, 131.7, 132.4, 132.4, 132.7, 137.3, 141.5, 141.8, 149.7; Anal. Calcd. for $\mathrm{C}_{23} \mathrm{H}_{25} \mathrm{NO}$ : C, 83.34; H, 7.60; N, 4.23\%. Found: C, 83.21; H, 7.54; $\mathrm{N}, 4.12 \%$.

2.2g 9-(2,6-Dichlorophenyl)-N-cyclohexylacenaphtho[1, 2-b]furan-8-amine (7g): Brown powder (92\%); m.p. 182-186 ${ }^{\circ}$; FT-IR $(\mathrm{KBr})\left(v_{\max }, \mathrm{cm}^{-1}\right): 3359(\mathrm{~N}-\mathrm{H})$; ${ }^{1} \mathrm{H}$ NMR $\left(400 \mathrm{MHz}, \mathrm{CDCl}_{3}\right) \delta_{\mathrm{H}} 1.22-2.00(10 \mathrm{H}, \mathrm{m}$, $\left.5 \mathrm{CH}_{2}\right), 3.52(1 \mathrm{H}, \mathrm{m}, \mathrm{N}-\mathrm{CH}), 6.22(1 \mathrm{H}, \mathrm{bs}, \mathrm{NH}), 7.23-$ $7.32(3 \mathrm{H}, \mathrm{m}, \mathrm{Ar}-\mathrm{H}), 7.60(2 \mathrm{H}, \mathrm{m}, \mathrm{Ar}-\mathrm{H}), 7.77-7.89$ $(4 \mathrm{H}, \mathrm{m}, \mathrm{Ar}-\mathrm{H}) ;{ }^{13} \mathrm{C}$ NMR $\left(100 \mathrm{MHz}, \mathrm{CDCl}_{3}\right) \delta_{\mathrm{C}} 25.5$, 31.3, 34.4, 52.5, 113.9, 129.6, 131.4, 131.6, 131.7, 132.4, 132.4, 133.1, 134.8, 136.7, 136.9, 138.7, 140.9, 142.5; Anal. Calcd. for $\mathrm{C}_{26} \mathrm{H}_{21} \mathrm{Cl}_{2} \mathrm{NO}$ : C, 71.89; $\mathrm{H}$, 4.87; N, 3.22\%. Found: C, 71.74; H, 4.81; N, 3.17\%.

2.2h N-tert-butyl-9-(2,4-dimethoxyphenyl)acenaphtho[1, 2-b]furan-8-amine (7h): Brown powder (95\%); m.p. $135-138^{\circ} \mathrm{C}$; FT-IR $(\mathrm{KBr})\left(\nu_{\max }, \mathrm{cm}^{-1}\right): 3364(\mathrm{~N}-\mathrm{H})$, 1210 (C-O), 1200 (C-O); ${ }^{1} \mathrm{H}$ NMR (400 MHz, $\left.\mathrm{CDCl}_{3}\right) \delta_{\mathrm{H}} 1.18\left(9 \mathrm{H}, \mathrm{s}, \mathrm{C}\left(\mathrm{CH}_{3}\right)_{3}\right), 3.70\left(3 \mathrm{H}, \mathrm{s}, \mathrm{OCH}_{3}\right)$, $3.74\left(3 \mathrm{H}, \mathrm{s},-\mathrm{OCH}_{3}\right), 5.41(1 \mathrm{H}, \mathrm{bs}, \mathrm{NH}), 6.59-6.69$ $(2 \mathrm{H}-\mathrm{m}, \mathrm{Ar}-\mathrm{H}), 7.19(1 \mathrm{H}, \mathrm{d}, \mathrm{Ar}-\mathrm{H}), 7.59-7.71(6 \mathrm{H}, \mathrm{m}$, $\mathrm{Ar}-\mathrm{H}) ;{ }^{13} \mathrm{C}$ NMR $\left(100 \mathrm{MHz}, \mathrm{CDCl}_{3}\right) \delta_{\mathrm{C}} 30.7,51.3$, $57.6,57.9,102.6,108.8,110.7,119.5,127.2,129$, 129.3, 129.7, 130.0, 130.7, 131.2, 135.2, 139.4, 140.7, 148.9, 160.1, 163.4; Anal. Calcd. for $\mathrm{C}_{26} \mathrm{H}_{25} \mathrm{NO}_{3}$ : C, 78.17; H, 6.31; N, 3.51\%. Found: C, 78.01; H, 6.24; N, $3.45 \%$.

2.2i N-tert-butyl-9-(4-nitrophenyl)acenaphtho[1,2b]furan-8-amine (7i): Brown powder (93\%); m.p. 146-148 ${ }^{\circ}$; FT-IR $(\mathrm{KBr})\left(v_{\max }, \mathrm{cm}^{-1}\right): 3364(\mathrm{~N}-\mathrm{H})$, $1550(\mathrm{~N}-\mathrm{O}), 1343(\mathrm{~N}-\mathrm{O}) ;{ }^{1} \mathrm{H}$ NMR $\left(400 \mathrm{MHz}, \mathrm{CDCl}_{3}\right)$ $\delta_{\mathrm{H}} 1.17\left(9 \mathrm{H}, \mathrm{s}, \mathrm{C}\left(\mathrm{CH}_{3}\right)_{3}\right), 6.43(1 \mathrm{H}, \mathrm{bs}, \mathrm{NH}), 7.75-7.80$ $(8 \mathrm{H}, \mathrm{m}, \mathrm{Ar}-\mathrm{H}), 8.29(2 \mathrm{H}, \mathrm{dd}, \mathrm{Ar}-\mathrm{H}) ; 7.76-8.30(10 \mathrm{H}$, $\mathrm{m}, \mathrm{Ar}-\mathrm{H}) ;{ }^{13} \mathrm{C}$ NMR $\left(100 \mathrm{MHz}, \mathrm{CDCl}_{3}\right) \delta_{\mathrm{C}} 31.8,53.9$, $113.1,123.7,127.5,128.1,130.0,130.3,131.5,132$, 132.7, 135.1, 137.5, 138.3, 139.8, 141.7, 143.9, 151.7, 157.2, 162.1, 177.8; Anal. Calcd. for $\mathrm{C}_{24} \mathrm{H}_{20} \mathrm{~N}_{2} \mathrm{O}_{3}$ : C, 74.98; H, 5.24; N, 7.29\%. Found: C, 74.71; H, 5.16; N, $7.19 \%$.
$2.2 \mathrm{j}$ 9-(2,4-Dimethoxyphenyl)- $N$-(2,6-dimethylphenyl) acenaphtho[1,2-b]furan-8-amine (7j): Brown powder (96\%); m.p. $163-167^{\circ} \mathrm{C}$; FT-IR (KBr) $\left(v_{\max }, \mathrm{cm}^{-1}\right)$ : $3451(\mathrm{~N}-\mathrm{H}), 1130(\mathrm{C}-\mathrm{O}), 1200(\mathrm{C}-\mathrm{O}) ;{ }^{1} \mathrm{H}$ NMR $\left(400 \mathrm{MHz}, \mathrm{CDCl}_{3}\right) \delta_{\mathrm{H}} 2.36(6 \mathrm{H}, \mathrm{s}, 2 \mathrm{Me}), 3.57(3 \mathrm{H}, \mathrm{s}$, $\left.\mathrm{OCH}_{3}\right), 3.62\left(3 \mathrm{H}, \mathrm{s},-\mathrm{OCH}_{3}\right), 6.74(1 \mathrm{H}, \mathrm{bs}, \mathrm{NH}), 6.43-$ $6.70(5 \mathrm{H}, \mathrm{m}, \mathrm{Ar}-\mathrm{H}), 7.26(1 \mathrm{H}, \mathrm{dd}, \mathrm{Ar}-\mathrm{H}), 7.60(2 \mathrm{H}, \mathrm{m}$, Ar-H), 7.69-7.84 (4H, m, Ar-H); ${ }^{13} \mathrm{C}$ NMR (100 MHz, $\left.\mathrm{CDCl}_{3}\right) \delta_{\mathrm{C}} 18.4,58.7,59.5,104.7,110.9,112.8,121.5$, 121.6, 128.4, 129.8, 130.2, 130.5, 130.9, 131.2, 131.8, 133.9, 134.4, 138.4, 142.6, 142.6, 144.8, 152.1, 163.3, 166.6; Anal. Calcd. for $\mathrm{C}_{30} \mathrm{H}_{25} \mathrm{NO}_{3}$ : C, 80.51; H, 5.63; N, 3.13\%. Found: C, 80.46; H, 5.58; N, 3.02\%.

\section{2k N-(2,6-Dimethylphenyl)-9-(4-nitrophenyl)} acenaphtho[1,2-b]furan-8-amine (7k): Brown powder $(94 \%)$; m.p. $142-144^{\circ} \mathrm{C}$; FT-IR $(\mathrm{KBr})\left(v_{\max }, \mathrm{cm}^{-1}\right)$ : $3237(\mathrm{~N}-\mathrm{H}), 1501(\mathrm{~N}-\mathrm{O}), 1418(\mathrm{~N}-\mathrm{O}) ;{ }^{1} \mathrm{H}$ NMR $\left(400 \mathrm{MHz}, \mathrm{CDCl}_{3}\right) \delta_{\mathrm{H}} 2.21(6 \mathrm{H}, \mathrm{s}, 2 \mathrm{Me}), 6.36(1 \mathrm{H}, \mathrm{bs}$, $\mathrm{NH}), 6.58(1 \mathrm{H}, \mathrm{dd}, \mathrm{Ar}-\mathrm{H}) 6.72(2 \mathrm{H}, \mathrm{m}, \mathrm{Ar}-\mathrm{H}), 7.66$ (2H, m, Ar-H), 7.74-7.90 (6H, m, Ar-H), $8.27(2 \mathrm{H}, \mathrm{d}$, $\mathrm{Ar}-\mathrm{H}) ;{ }^{13} \mathrm{C}$ NMR $\left(100 \mathrm{MHz}, \mathrm{CDCl}_{3}\right) \delta_{\mathrm{C}} 22.4,113.1$, $122.7,125.7,129.6,129.7,130.5,130.4,130.7,130.8$, 131.9, 134.0, 134.1, 138.6, 142.2, 142.8, 145.2, 147.6, 152.3, 153.5; Anal. Calcd. for $\mathrm{C}_{28} \mathrm{H}_{20} \mathrm{~N}_{2} \mathrm{O}_{3}$ : C, 77.76; H, 4.66; N, 6.48\%. Found: C, 77.58; H, 4.43; N, 6.41\%.

\section{Results and discussions}

Three ionic liquid catalysts $(10 \% \mathrm{~mol})$ of $[\mathrm{pmim}] \mathrm{Cl}$ (1-propyl-3-methylimidazolium-Cl) (a), $\left[\mathrm{pmim} \mathrm{BF}_{4}\right.$ (1-propyl-3-methylimidazolium- $\mathrm{BF}_{4}$ ) (b) and $\left[\mathrm{pmim} \mathrm{HSO}_{4}\right.$ (1-propyl-3-methylimidazolium- $\mathrm{HSO}_{4}$ ) (c) (scheme 1), possessing different counteranions, were evaluated for catalysing the reaction of an equal stoichiometric ratio of silyl enol of acenaphthylen-1(2H)-one (1), 2,4dimethoxybenzaldehyde and isocyanocyclohexane. As a result, catalyst (c) was found as the best choice (table 2).

In order to reveal the plausible effect of supporting on the catalytic behaviour of $[\mathrm{pmim}] \mathrm{HSO}_{4}(\mathbf{c})$, it was immobilized on the modified silica. The use of supported catalyst, [pmim] $\mathrm{HSO}_{4}-\mathrm{SiO}_{2}$ (d) (scheme 1), resulted in a significant improvement in reaction times and catalytic yields attributed plausibly to the participation of $\mathrm{SiO}_{2}$ in the catalytic process (table 2, entry 2). However, this hypothesis was established to some extent when the silica support was used as catalyst under the same reaction conditions. As can be in table 2 (compare entries 1 and 6), efficiencies of the reactions 
Table 2. Catalytic efficiencies of ionic liquids (a-d) for the preparation of compound $(7 \mathbf{a}){ }^{\mathrm{a}}$

\begin{tabular}{llcc}
\hline Entry & \multicolumn{1}{c}{ Catalyst } & Time (h) & Yield (\%) \\
\hline 1 & ----- & 24 & 89 \\
2 & {$\left[\mathrm{pmim}^{\mathrm{b}}\right] \mathrm{HSO}_{4}-\mathrm{SiO}_{2}$} & 10 & 95 \\
3 & {$[\mathrm{pmim}] \mathrm{HSO}_{4}$} & 14 & 92 \\
4 & {$[\mathrm{pmim}] \mathrm{BF}_{4}$} & 18 & 90 \\
5 & {$[\mathrm{pmim}] \mathrm{Cl}$} & 18 & 88 \\
6 & Silica support & 20 & 87 \\
\hline
\end{tabular}

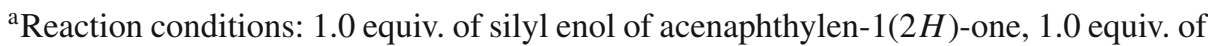
2,4-dimethoxybenzaldehyde, 1.0 equiv. of isocyanocyclohexane, $10 \mathrm{~mol} \%$ of catalyst, $60 \mathrm{~mL}$ of DMF as solvent and at refluxing condition.

b'Isolated yields

in terms of times and yields compared with uncatalysed system were found better. So, supported ionic liquid (d) was used to assess the generality and scope of reaction with respect to the aldehyde and isocyanide components (table 1). As a result, the reactions proceeded well with various aromatic and aliphatic aldehydes, such as $\alpha, \beta$-unsaturated aldehydes, and electronically sufficient and deficient aldehydes. Moreover, employing sterically hindered isocyanides also could not prevent the reactions to afford their good yields (table 1, entries 8-11). As can be seen, all chemical yields and reaction times were improved in the presence of ionic liquid (d) compared with those we obtained under uncatalysed conditions.

With the aim of investigating the effects of temperature and solvent, the reactions were carried out at the various temperatures and in the different solvents such as $\mathrm{CH}_{2} \mathrm{Cl}_{2}, \mathrm{CHCl}_{3}$, ethanol, $\mathrm{CH}_{3} \mathrm{CN}$, and finally refluxed DMSO were found as the best reaction conditions.

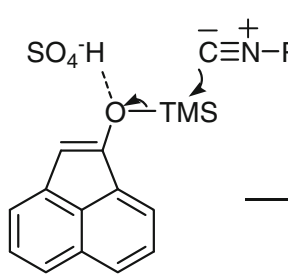

(1)<smiles>[R]Nc1oc2c(c1[R])-c1cccc3cccc-2c13</smiles>

(7a-k)<smiles>C[Si](C)OC1=Cc2cccc3cccc1c23</smiles>

(2)

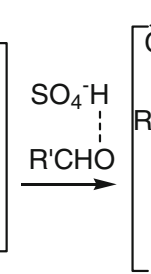

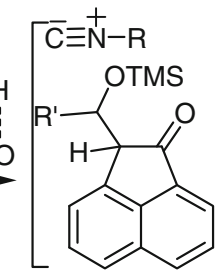

(3)

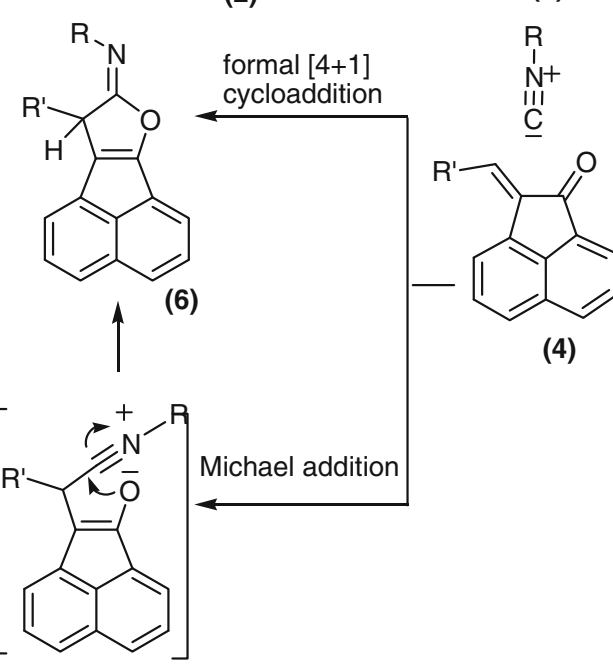

(5)<smiles>CCCC</smiles>

- TMSOH

Scheme 2. Proposed mechanism for the one-pot three-component synthesis of compounds 7a-k. 
As established in our previous studies, ${ }^{21,22}$ at the first step, isocyanide attacks on $\mathrm{O}-\mathrm{SiMe}_{3}$ bond of silyl enol ether (1) to generate active enol ion pair complex (2). Then, $\alpha, \beta$-unsaturated ketone (4) generated after condensation of enol (2) and aldehyde can produce iminolactone intermediate (6) either via a formal [4+1]-cycloaddition reaction or a Michael-type addition with the isocyanide. Finally, the intermediate (6) isomerizes to form aminofuran heteroaromatic moiety (7) (scheme 2).

\section{Conclusion}

A novel multicomponent approach for the synthesis of a series of new acenaphthofuran derivatives utilizing the supported ionic liquid catalyst has been elaborated. The efficient catalysing of used ionic liquid in the synthesis of acenaphtho[1,2- $b]$ furans led to high chemical yields as well as short reaction times.

\section{References}

1. Dean F M 1982 Adv. Hetero. Chem. 30167

2. Lipshutz B H 1986 Chem. Rev. 795

3. Sternbach D D and Rossana D M 1982 Tetrahedron Lett. 23303

4. Baghernejad B, Heravi M M, Oskooie $\mathrm{H}$ A, Poormohammad N, Khorshidi M and Beheshtiha Y S 2011 Mol. Div. 15245
5. Teimouri M B and Mansouri F 2008 J. Comb. Chem. 10 507

6. Shaabani A, Rezayan A H, Ghasemi S and Sarvary A 2009 Tetrahedron Lett. 501456

7. Zhu X, Xu X P, Sun C, Chen T, Shen Z L and Ji S J 2011 Tetrahedron 676375

8. Passerini M 1922 Gazz. Chim. Ital. 52432

9. Bienaymé H, Hulme C, Oddon G and Schmitt P 2000 Chem. Eur. J. 63321

10. Orru R V A and De Greef M 2003 Synthesis 1471

11. Von Wangelin A J, Neumann H, Gördes D, Klaus S, Strübing D and Beller M 2003 Chem. Eur. J. 94286

12. Ugi I and Steinbrückner C 1960 Angew. Chem. 72267

13. Ugi R, Meyr I, Fetzer U and Steinbrückner C 1959 Angew. Chem. 71386

14. Ugi I, Lohberger S and Karl R 1991 Comprehensive Org. Synth. 21083

15. Wasserscheid P and Welton T 2007 Ionic liquids in synthesis, 2nd edn., VCH, Einheim: Wiley

16. Du Y, Tian F and Zhao W 2006 Synth. Commun. 36 1661

17. Gupta N, Kad G L and Singh J 2007 Catal. Commun. 8 1323

18. Hajipour A R, Rajaei A and Ruoho A E 2009 Tetrahedron Lett. $\mathbf{5 0} 708$

19. Khosropour A R 2008 Can. J. Chem. 86264

20. Wang W, Cheng W, Shao L and Yang J 2008 Catal. Lett. 12177

21. Goldani M T, Sandaroos R and Damavandi S 2012 Chin. Chem. Lett. 23169

22. Sandaroos R, Damavandi S, Salimi M, Mohammadi A, Karimian A and Hasanpour 2012 Mol. Divers. 16269

23. Song J J, Tan Z, Reeves J T, Fandrick D R, Yee N K and Senanayake C H 2008 Org. Lett. 10877

24. Sandaroos R, Damavandi S and Salimi M 2012 Monatsh Chem., DOI 10.1007/s00706-012-0744-2 